\title{
Painless Presentation of a Deadly Disease: Type A Aortic Dissection Requiring the Bentall Procedure
}

\author{
Muhammad Atif Masood Noori ${ }^{1}$, Kalpesh Shah², Hardik Fichadiya ${ }^{1}$, Mofe Adeosun $^{3}$, Shrutie Jesani ${ }^{1}$, Edmund Appiah-Kubi $^{3}$, \\ Hasham Saeed ${ }^{1}$, Sherif Elkattawy ${ }^{1}$, Meherwan Joshi ${ }^{1}$ \\ ${ }^{1}$ Department of Medicine, Rutgers Health/Trinitas Regional Medical Center, Elizabeth, NJ, USA \\ ${ }^{2}$ Department of Cardiology, St. Joseph's University Medical Center, Paterson, NJ, USA \\ ${ }^{3}$ St. George's University, True Blue, Grenada
}

Received: $17 / 01 / 2022$

Accepted: 01/02/2022

Published: 01/03/2022

How to cite this article: Noori MAM, Shah K, Fichadiya H, Adeosun M, Jesani S, Appiah-Kubi E, Saeed H, Elkattawy S, Joshi M. Painless presentation of a deadly disease: type A aortic dissection requiring the Bentall procedure. EJCRIM 2022;9:doi:10.12890/2022_003197.

Conflicts of Interests: The Authors declare that there are no competing interests.

This article is licensed under a Commons Attribution Non-Commercial 4.0 License

\section{ABSTRACT}

Aortic dissection is a relatively uncommon, although catastrophic, disease which requires early and accurate diagnosis and treatment for patient survival. Aortic dissection can be difficult to diagnose due to the diverse symptom presentation, which can lead to later diagnosis, resulting in a higher mortality rate. Here we present a case of type A aortic dissection with a varied symptom presentation, highlighting the importance of early detection and the Bentall procedure for management of such cases. A 50-year-old man with no known medical history presented with bilateral lower extremity swelling and fatigue for 2 weeks. The patient denied any chest pain or dyspnoea. Vital signs showed blood pressure of $160 / 76 \mathrm{mmHg}$, pulse of $103 \mathrm{bpm}$, respiratory rate of 18 , and temperature of $36.7^{\circ} \mathrm{C}$. Laboratory findings indicated a BNP of $1901 \mathrm{pg} / \mathrm{ml}$ and troponin of $0.5 \mathrm{ng} / \mathrm{ml}$. An initial diagnosis of decompensated heart failure was made, and IV Lasix was started. Subsequently, an echocardiogram indicated an EF of 50-55\% and ascending dissection of the aorta. A CT angiogram of the chest and abdomen confirmed this diagnosis. This patient presented with unusual symptoms of aortic dissection without the typical presentation of chest pain. It is important to consider aortic dissection in a cardiac-related case as prompt imaging can help confirm the diagnosis. We explore the risks and benefits of the Bentall procedure for the management and early detection of aortic dissection.

\section{KEYWORDS}

Type A aortic dissection, Bentall procedure, atypical symptoms

\section{LEARNING POINTS}

- The absence of chest pain does not rule out aortic dissection as $50 \%$ of patients are pain free.

- It is critical to diagnose aortic dissection early in the disease course as mortality increases by $1 \%$ per hour from symptom onset.

- The aim of this study is also to raise awareness among healthcare professionals about the Bentall procedure in patients with type A aortic dissection involving the aortic valve.

\section{INTRODUCTION}

Aortic dissection is a challenging disease to diagnose as its presenting symptoms are so diverse. If not diagnosed when symptoms begin, its mortality rate is $1 \%$ per hour, so early and accurate diagnosis is very important. 
There are two types of aortic dissection: Stanford type A and Stanford type B, depending on the involvement of the ascending aorta. Stanford type A involves the ascending aorta and accounts for $60 \%$ of aortic dissections, while type B occurs distal to the left subclavian artery. Type A aortic dissection is misdiagnosed in $40 \%$ of cases due to overlap with acute coronary symptoms ${ }^{[1]}$. Type A presents with a higher mortality rate and therefore requires prompt surgical treatment and management ${ }^{[1]}$.

We present a case of type A aortic dissection in a middle-aged man without typical presenting symptoms in order to highlight the importance of using the Bentall procedure and to encourage a high index of suspicion in patients with atypical symptoms.

\section{CASE DESCRIPTION}

A 50-year-old man with no known medical history presented to the emergency room with complaints of bilateral leg swelling associated with fatigue for the past 2 weeks. The patient denied any chest pain, shortness of breath, orthopnoea, paroxysmal nocturnal dyspnoea, fever, palpitations and change in urinary or bowel habits. On arrival, the patient's blood pressure was $160 / 76 \mathrm{mmHg}$, pulse rate was 103 bpm, respiratory rate was 18 , and temperature $36.7^{\circ} \mathrm{C}$. Chest $x$-ray showed hazy interstitial opacities throughout the lung fields suggestive of pulmonary oedema. Laboratory studies showed the following values: WBC count $8.1 \mathrm{~K} / \mu \mathrm{l}$, Hgb $10.7 \mathrm{~g} / \mathrm{dl}$, PLT $338 \mathrm{~K}$, creatinine 0.86 $\mathrm{mg} / \mathrm{dl}$, BUN $16 \mathrm{mg} / \mathrm{dl}$, sodium $141 \mathrm{mmol} / \mathrm{l}$, potassium $4.5 \mathrm{mmol} / \mathrm{l}$, BNP PoCT $1901 \mathrm{pg} / \mathrm{ml}$ and troponin $0.05 \mathrm{ng} / \mathrm{ml}$. A diagnosis of acute decompensated heart failure was made, and the patient was started on IV Lasix $40 \mathrm{mg}$ twice a day with losartan $50 \mathrm{mg}$ daily.

Next day, a transthoracic echocardiogram showed an LVEF of 50-55\% but also revealed dissection of the ascending aorta, the arch and descending aorta with an enlarged aortic root and moderate to severe aortic insufficiency (Figs. 1-3). To confirm the 2D echo findings, a CT angiogram of the chest and abdomen was done which showed Stanford type A aortic dissection extending from the proximal ascending aorta to the distal aorta with extension into the common iliac and external iliac arteries (Figs. 4 and 5). The patient was transferred to ICU where a metoprolol and nicardipine drip was started to keep the heart rate and systolic blood pressure below 60 and 120 , respectively. Cardiothoracic surgery was consulted, and the patient was transferred to another facility for aortic dissection repair where he underwent the Bentall procedure and aortic valve replacement.

After the procedure, the patient was transferred back to our facility for continuity of care. A repeat 2D echo showed an LVEF of 45-50\%. Goal-directed medical therapy for congestive heart failure was continued and after the therapeutic level of INR was reached for aortic valve replacement, the patient was discharged to follow-up with cardiology as an outpatient.

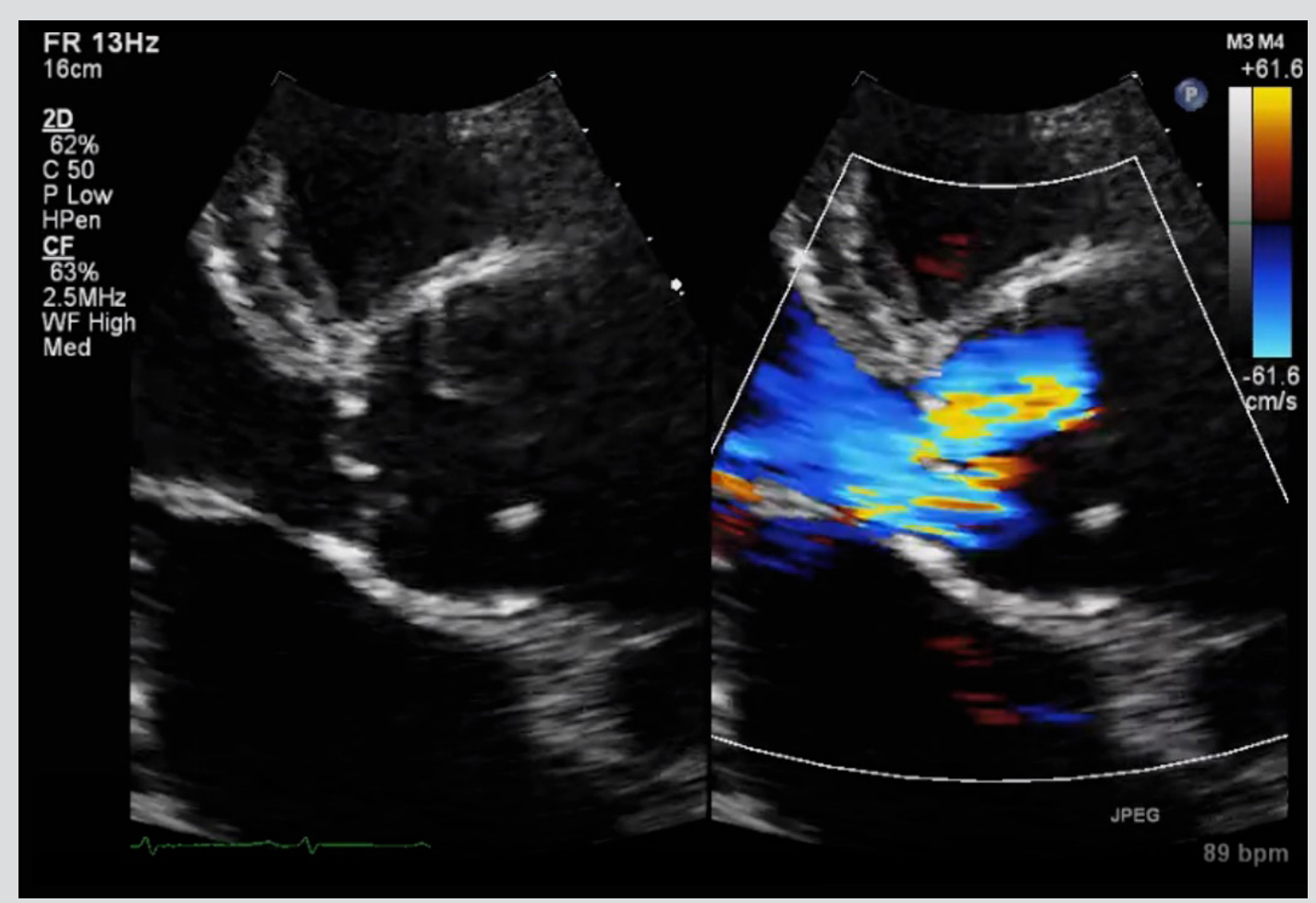

Figure 1. Parasternal long axis view: the left side of the image shows a dissection flap and enlarged aortic root, and the right side shows aortic regurgitation 

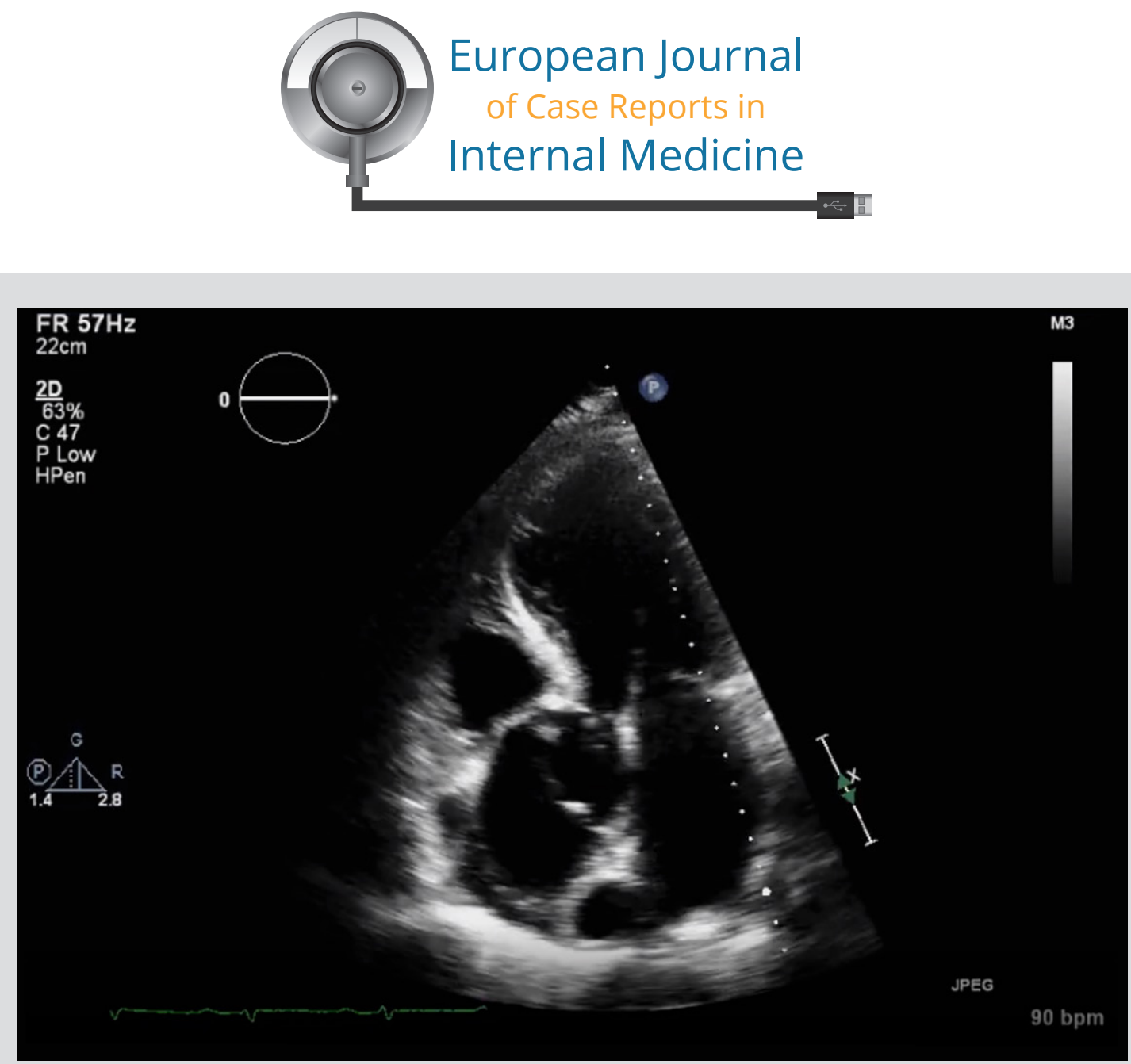

Figure 2. Apical five-chamber view showing a dissection flap in the aorta

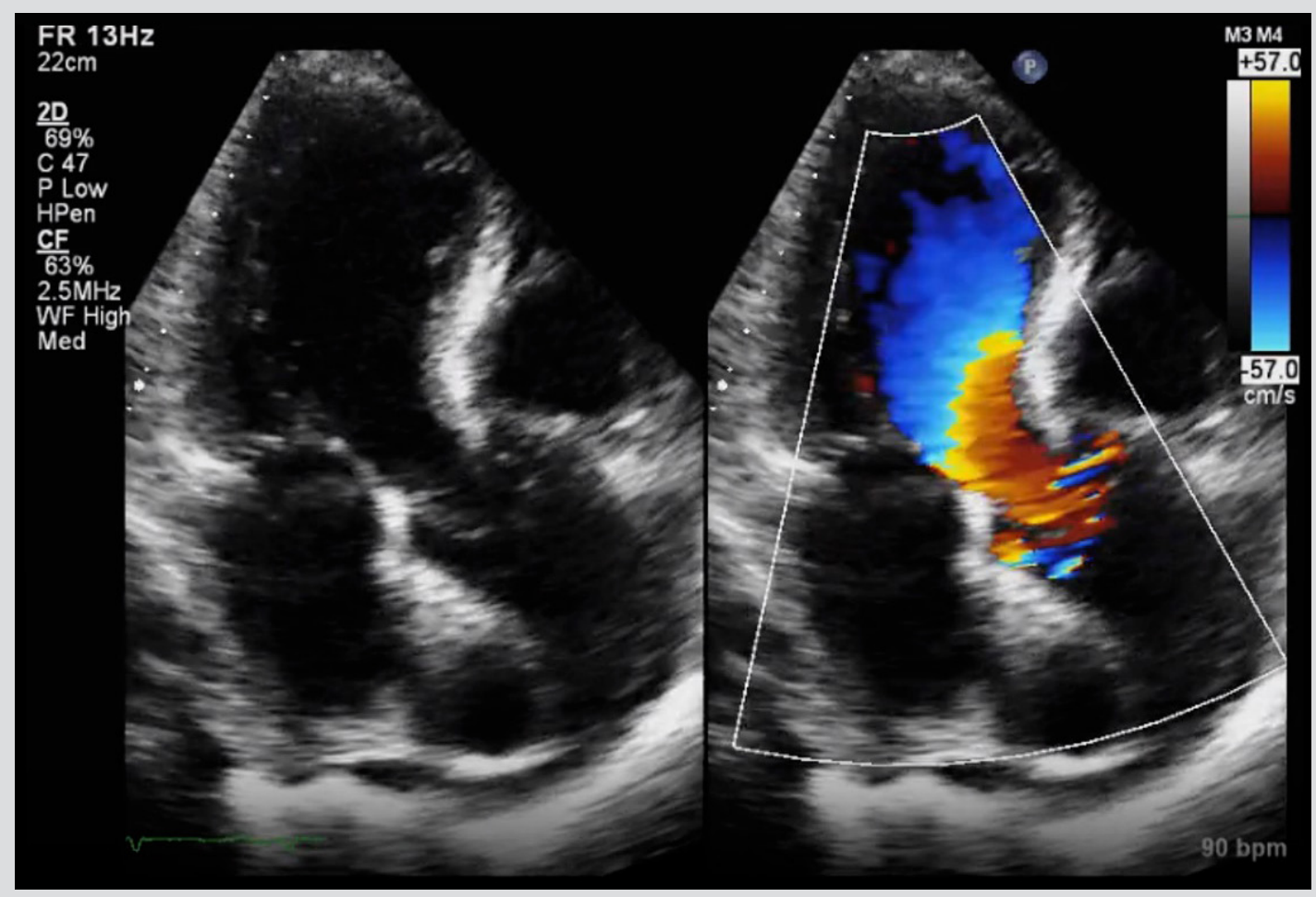

Figure 3. Apical five-chamber view showing aortic regurgitation 


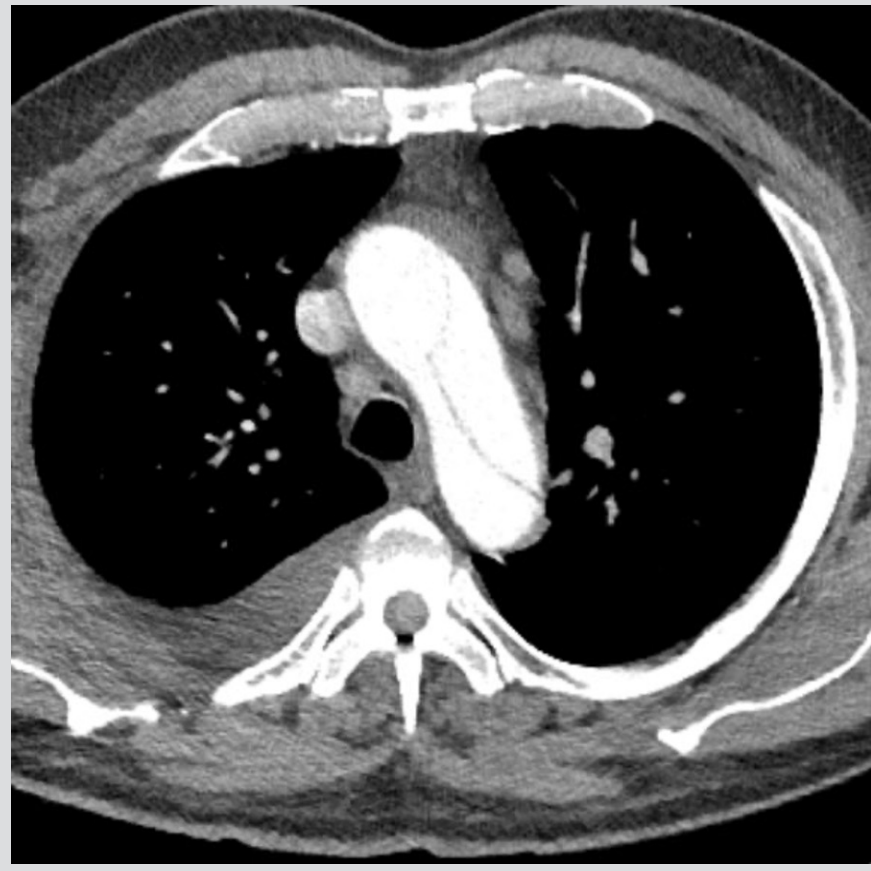

Figure 4. CT angiogram of the chest showing a dissection flap in the aortic arch and descending aorta

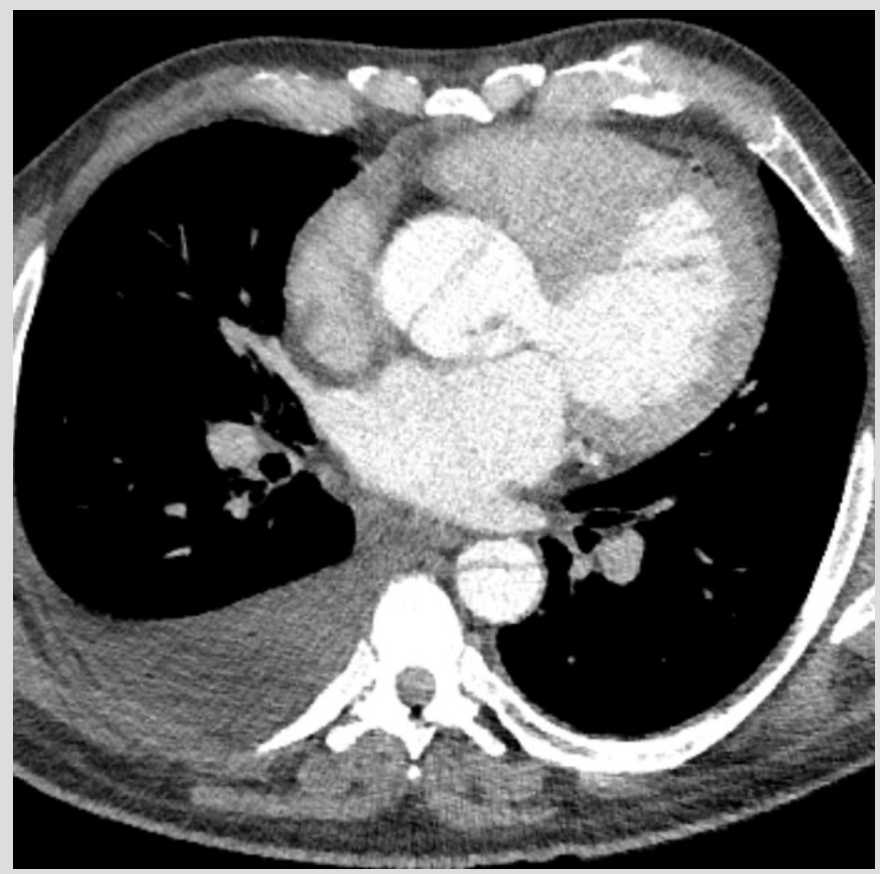

Figure 5. CT angiogram of the chest showing a dissection flap in the ascending aorta

\section{DISCUSSION}

Aortic dissection type $\mathrm{A}$ has a diverse symptom presentation. This case is unique as it highlights: (a) that the signs of aortic dissection may not include the atypical chest pain; (b) the role of echocardiography and CT in the prompt diagnosis of aortic dissection; and (c) the use of the Bentall procedure to repair aortic dissection.

When assessing a patient with suspected aortic dissection, the quality, radiation and intensity of pain should be considered. The most common presenting complaint is sudden-onset tearing chest pain radiating to the back, associated with a drop in blood pressure or pulse deficit in the upper limbs ${ }^{[1]}$. These symptoms are present in fewer than $50 \%$ of confirmed cases of acute aortic dissection (AAD) ${ }^{[2]}$. The presence of chest pain in addition to neurological findings, abdominal pain, limb weakness and paraesthesia should alert the physician to the possibility of aortic dissection. Other physical findings in patients with AAD include hypertension, aortic insufficiency, syncope and muffled heart sounds ${ }^{[2]}$. AAD is frequently mistaken for other disorders that cause chest pain, the most common being acute coronary syndromes. The absence of chest pain and the presentation of syncope, vascular insufficiency and gastrointestinal symptoms may lead to the misdiagnosis of aortic dissection. The incidence of misdiagnosis is $40 \%$ and is commonly associated with the ascending aorta. Errors in misdiagnosis delay proper treatment, leading to a further risk of complications ${ }^{[1]}$. It is important for medical professionals to have a high index of suspicion for patients presenting with risk factors and atypical symptoms ${ }^{[3,4]}$.

Some $40 \%$ of patients above 40 years of age do not present with the typical features and characteristics ${ }^{[4]}$. In our case, the patient was a middle-aged man presenting with oedema and fatigue but no chest pain. He had an elevated blood pressure and pulse rate. Arterial hypertension is a major risk factor $(77.8 \%)$ for $A A D^{[4]}$. The patient's chest $x$-ray showed hazy interstitial opacities throughout the lung fields, which indicates decompensated heart failure. If diagnosis had ended here, there would have been a misdiagnosis as chest $x$-ray has a sensitivity of $67 \%$ and specificity of $70 \%{ }^{[4]}$. However, an echocardiogram was also obtained, and it showed an LVEF of $50-55 \%$. It also showed dissection of the ascending aorta. To further confirm the diagnosis, a CT angiogram of the chest and abdomen was done which revealed Stanford type A aortic dissection.

The Bentall procedure was carried out the next day to treat the aortic dissection. This procedure consists of surgical repair of an ascending aortic or aortic root aneurysm in combination with aortic valve disease. A composite aortic valve graft is used to replace the proximal ascending aorta and aortic valve ${ }^{[5]}$. The replacement valve can be mechanical or prosthetic. The Bentall procedure is the ideal surgery for all acute type A dissections in circumstances when the aortic root is dilated more than $4.5 \mathrm{~cm}$ and presents with an intimal tear or connective tissue disease. The Bentall procedure has disadvantages as it requires lifelong anticoagulation, and has an increased risk of stroke, bleeding, 
infectious endocarditis and haemolysis ${ }^{[6]}$. An aortic valve sparing procedure is recommended for younger patients below the age of 40 due to bioprosthetic valve degeneration and the requirement for lifelong anticoagulation therapy, while those above 40, like the patient in our case, are offered the option of a mechanical valve and stented bioprosthesis ${ }^{[6,7]}$. In a study by Yang et al., 135 patients with type A aortic dissection were followed for 16 years between 2001 and 2017 to observe the outcomes of Bentall and David procedures. Following the Bentall procedure in a middle-aged population, the mortality rate was $13 \%$, the rate of reoperation of the proximal aorta or aortic valve was $2 \%$, and the 10 -year survival rate was $57 \%[6]$.

\section{CONCLUSIONS}

Type A aortic dissection is a devastating disorder with multiple presentations. Misdiagnosis due to the absence of typical features, especially chest pain, can result in later intervention and increased mortality. A high index of suspicion is required when type A aortic dissection presents atypically, requiring eventual treatment with the Bentall procedure. This procedure is the standard treatment and has a favourable prognosis in middle-aged patients. We hope this case report highlights the less common presentations of aortic dissection and informs clinicians of the steps which can be taken to ensure the safety of the patient. Hopefully, further research on the different surgical procedures for treating aortic dissection will be carried out and help improve patient outcomes.

\section{REFERENCES}

1. Ahmed T, Nair R, Lak H, Lodhi SH, Maroo A. Two intriguing cases of Stanford type A acute aortic dissection. Cureus 2020;12(2):e6986. doi: 10.7759/CUREUS.6986

2. Levy D, Goyal A, Grigorova Y, Farci F, Le JK. Aortic dissection. StatPearls. Published online 17 June 2021 (accessed 24 December 2021 ). Available from https://www.ncbi.nlm. nih.gov/books/NBK441963/

3. Mando R, Tim D, DeCicco A, Trivax J, Hanson I. Master of the masquerade: an atypical presentation of acute aortic dissection. Case Rep Cardiol 2020;2020:5743985. doi: $10.1155 / 2020 / 5743985$

4. Solhjoo M, Swarup S, Makaryus AN. A case of aortic dissection presenting with atypical symptoms and diagnosed with transthoracic echocardiography. Case Rep Radiol 2019;2019:6545472. doi: 10.1155/2019/6545472

5. Cherry C, DeBord S, Hickey C. The modified Bentall procedure for aortic root replacement. AORN J 2006;84(1):52-55, 58-70. doi: 10.1016/S0001-2092(06)60098-7

6. Yang B, Patel HJ, Sorek C, Hornsby WE, Wu X, Ward S, et al. Sixteen-year experience of David and Bentall procedures in acute type A aortic dissection. Ann Thorac Surg 2018;105(3):779-784. doi: 10.1016/J.ATHORACSUR.2017.09.029

7. Etz CD, Bischoff MS, Bodian C, Roder F, Brenner R, Griepp RB, et al. The Bentall procedure: is it the gold standard? A series of 597 consecutive cases. J Thorac Cardiovasc Surg 2010;140:S64-S70. doi: 10.1016/j.jtcvs.2010.07.033 